\title{
ON THE PARTNERSHIP FORMATION PROBLEM
}

\author{
Akiyoshi Shioura \\ Tokyo Institute of Technology, Japan \\ shioura.a.aa@m.titech.ac.jp
}

\begin{abstract}
In this paper we re-examine the partnership formation problem, which is a generalization of the classical assignment game. We show that the former problem can be transformed into the latter one in some sense; more precisely, we demonstrate that using an equilibrium in an associated assignment game, we can find an equilibrium in the partnership formation problem if it exists. Based on this, we devise an algorithm to compute an equilibrium of the partnership formation problem, and show that the proposed algorithm can be seen as a variant of the one by Andersson et al. (2014b).

Keywords: Partnership formation, equilibrium, assignment game, adjustment process.

JEL Classification Numbers: C71, D44.
\end{abstract}

\section{INTRODUCTION}

In this paper we reconsider the partnership formation problem as studied by Talman \& Yang (2011). In such a problem, there is a group of agents, and each agent works alone or works together with another agent. If an agent works alone, then the agent generates a value for himself, and if an agent works with a partner, then the agent and his partner generate a joint value, which is shared by them in an appropriate way. The goal of the partnership formation problem is to find an equilibrium, where no agent has incentive to change his partner, to break up an existing partnership to become alone,

The author thanks Kazuo Murota and Zaifu Yang for their valuable comments on the manuscript. This work is supported by JSPS/MEXT KAKENHI Grand Numbers 15K00030, $15 \mathrm{H} 00848$.

Copyright (c) Akiyoshi Shioura / 2(1), 2017, 105-140. 
or to form a new partnership. Typical instances of the partnership formation problem can be found in the professional tennis tournament, pair programming in software development, etc. (see, e.g., Andersson et al. (2014b); Eriksson \& Karlander (2001); Talman \& Yang (2011)). Similar but different models of the partnership formation are also discussed in Chiappori et al. (2014); Alkan \& Tuncay (2013). The partnership formation problem has been also formulated as the roommate problem with transferable utility, the matching game, or the one-sided matching problem (Eriksson \& Karlander, 2001; Biró et al., 2012; Klaus \& Nichifor, 2010).

The partnership formation problem is closely related to the classical assignment game (Koopmans \& Beckmann, 1957; Shapley \& Shubik, 1971). The assignment game can be regarded as a special case of the partnership formation problem, where the set of agents is partitioned into two groups, the one corresponding to buyers (or firms) and the other to sellers (or workers), and any two agents in the same group cannot be a pair. An equilibrium always exists in the assignment game (Koopmans \& Beckmann, 1957; Shapley \& Shubik, 1971), and can be found by price adjustment processes (see, e.g., Crawford \& Knoer (1981), Demange et al. (1986)).

In contrast, the partnership formation problem may not have an equilibrium (Talman \& Yang, 2011). Some sufficient (and necessary) conditions for the existence of equilibrium are provided by Eriksson \& Karlander (2001) and Talman \& Yang (2011). An adjustment process is proposed for the partnership formation problem by Andersson et al. (2014b), which can always either find an equilibrium or disprove the existence of an equilibrium. The adjustment process of Andersson et al. (2014b) computes a certain payoff vector in a similar way as in the adjustment process by Demange et al. (1986), and the obtained payoff vector is used to find an equilibrium of the partnership formation problem.

The main aim of this paper is to clarify the relationship between the partnership formation problem and the assignment game. As mentioned above, the assignment game is a very special case of the partnership formation problem. We show in this paper that the converse is also true in some sense. That is, we prove that if we obtain an equilibrium in a certain assignment game, then we can find an equilibrium in the partnership formation problem or disprove the existence of an equilibrium.

For this, we associate an assignment game with a given partnership formation problem in Section 3.1, and show in Sections 3.2 and 3.3 that the 
associated assignment game has various properties that are useful in finding an equilibrium of the partnership formation problem. In particular, we show that an equilibrium in the partnership formation problem corresponds to an equilibrium in the associated assignment game satisfying a certain condition (Theorems 3.3 and 3.4). Then, it is shown that using an equilibrium payoff in the associated assignment game, the problem of finding an equilibrium in the partnership formation problem can be reduced to the problem of finding a matching among the agents such that each agent is matched to one of his favorite agents (Theorem 3.5). Base on this property, we devise an algorithm for computing an equilibrium of the partnership formation problem.

It is observed that our algorithm is similar to the algorithm by Andersson et al. (2014b). Indeed, the starting point of our current research is to better understand the behavior of their algorithm. In Section 4 we discuss the connection between our algorithm and the one by Andersson et al. (2014b), and show that our algorithm can be seen as a variant of the algorithm by Andersson et al. (2014b) with more flexibility.

We finally note that our approach by using an associated assignment game is not totally new, and similar approach is already used in Biró et al. (2012) and Chiappori et al. (2014). Indeed, Biró et al. (2012) and Chiappori et al. (2014) associate certain assignment games with a given partnership formation problem, and use the maximum weight of a matching in the associated assignment games to characterize the existence of an equilibrium in the partnership formation problem. Based on the characterization, Biró et al. (2012) and Chiappori et al. (2014) propose algorithms for checking the existence of an equilibrium in partnership formation problem. A drawback of their algorithms is that the information about the joint values of agents pairs are needed to compute the maximum weight of a matching.

In contrast, the associated assignment game used in this paper is similar to but different from the ones in Biró et al. (2012) and Chiappori et al. (2014) (see Section 3.1 for more discussion on the difference of the associated assignment games). This difference makes it possible to obtain a characterization for the existence of an equilibrium in terms of demand correspondences of agents. This characterization is useful in designing an adjustment process for checking the existence of an equilibrium, which does not require the information about the joint values of agents pairs. 


\section{PRELIMINARIES}

We review definitions and fundamental properties for the partnership formation problem and the assignment game. In the following, we denote by $\mathbb{Z}_{+}$and $\mathbb{R}_{+}$ the sets of non-negative integers and non-negative real numbers, respectively.

\subsection{Partnership Formation Problem}

We explain the model of the partnership formation problem in Biró et al. (2012), which is (slightly) more general than the original one in Talman \& Yang (2011).

An instance of the partnership formation problem is given by a tuple $(N, E, v)$, where $(N, E)$ is an undirected graph and $v: E \rightarrow \mathbb{R}_{+}$is an edge weight function taking non-negative real numbers. The vertex set $N$ represents a set of agents, where it is assumed that there are $n$ agents and $N=\{1,2, \ldots, n\}$. Each agent works alone or works together with another agent. We consider the setting where possible partners of agents are restricted for some reasons such as their skills and/or human relationship, and possible pairs are represented by the edge set $E \subseteq\{(i, j) \mid i, j \in N, i \neq j\}$. That is, two agents $i$ and $j$ can work together if and only if $(i, j) \in E$. The original model of Talman \& Yang (2011) corresponds to the case where any two agents can be a pair, i.e., $E=\{(i, j) \mid i, j \in N, i \neq j\}$. We note that $(i, j) \in E$ if and only if $(j, i) \in E$ for every $i, j \in N$. For $(i, j) \in E$, the edge weight $v(i, j)$ represents the (joint) value generated by the two agents $i$ and $j$. We assume, without loss of generality, that the value generated by a single agent $i \in N$ is equal to zero.

A vector $p=\left(p_{1}, p_{2}, \ldots, p_{n}\right) \in \mathbb{R}^{N}$ is called a payoff. A matching is a function $\mu: N \rightarrow N$ such that for $i, j \in N$, we have $\mu(i)=j$ if and only if $\mu(j)=i$. For $i \in N$, if $\mu(i) \neq i$ then agent $\mu(i)$ is the partner of agent $i$ in the matching $\mu$, while $\mu(i)=i$ means that agent $i$ has no partner in the matching $\mu$. Therefore, a matching corresponds to a partition of $N$ into pairs of agents and single agents.

A pair of a matching $\mu$ and a payoff $p$ is called an equilibrium in the Journal of Mechanism and Institution Design 2(1), 2017 
partnership formation problem $(N, E, v)$ if the following conditions hold: ${ }^{1}$

$$
\begin{array}{ll}
p_{i}+p_{j} \geq v(i, j) & (\forall(i, j) \in E), \\
p_{\mu(i)}+p_{i}=v(\mu(i), i) & (\forall i \in N \text { with } \mu(i) \neq i), \\
p_{i} \geq 0 & (\forall i \in N), \\
p_{i}=0 & (\forall i \in N \text { with } \mu(i)=i) .
\end{array}
$$

An equilibrium in the partnership formation problem may not exist (see, e.g., Chiappori et al. (2014); Talman \& Yang (2011)). For example, it is easy to see that the partnership formation problem with $N=\{1,2,3\}, E=$ $\{(1,2),(2,3),(1,3)\}$, and $v(i, j)=1$ for every $(i, j) \in E$ has no equilibrium.

A matching (resp., a payoff) in an equilibrium is called an equilibrium matching (resp., an equilibrium payoff). The partnership formation problem is called the matching game in Biró et al. (2012), where the set of equilibrium payoffs in the partnership formation problem is called the core, while an equilibrium payoff is called a core allocation.

The conditions (2.1)-(2.4) for an equilibrium can be simply rewritten as follows:

$$
\begin{array}{ll}
p_{i}+p_{j} \geq v(i, j) & (\forall(i, j) \in \bar{E}), \\
p_{\mu(i)}+p_{i}=v(\mu(i), i) & (\forall i \in N),
\end{array}
$$

where

$$
v(i, i)=0 \quad(i \in N), \quad \bar{E}=E \cup\{(i, i) \mid i \in N\} .
$$

Note that the pair $(N, \bar{E})$ can be seen as an undirected graphs with a self-loop at each vertex.

The conditions (2.5) and (2.6) can be further rewritten in terms of demand correspondences. For an agent $i \in N$ and a payoff $p \in \mathbb{R}^{N}$, we define the demand correspondence $D_{i}(p) \subseteq N$ as the set of agents $j \in N$ that maximize $v(i, j)-p_{j}$, i.e.,

$$
D_{i}(p)=\arg \max \left\{v(i, j)-p_{j} \mid j \in N,(i, j) \in \bar{E}\right\} .
$$

We consider the following two conditions, which are shown to be equivalent to (2.5) and (2.6); the first condition means that the partner of each agent is one of his favorite agents, and the second condition means that the payoff of

1 The symbol " $\forall$ " reads "for all" or "for every." 
each agent $i \in N$ is equal to the maximum of the value $v(i, j)-p_{j}$ among all possible partners $j \in N$.

$$
\begin{aligned}
& \mu(i) \in D_{i}(p) \quad(\forall i \in N), \\
& p_{i}=\max \left\{v(i, j)-p_{j} \mid j \in N,(i, j) \in \bar{E}\right\} \quad(\forall i \in N) .
\end{aligned}
$$

Proposition 2.1. Let $\mu: N \rightarrow N$ and $p \in \mathbb{R}^{N}$ be a matching and a payoff, respectively, in the partnership formation problem $(N, E, v)$. Then, the pair $(\mu, p)$ is an equilibrium in $(N, E, v)$ if and only if it satisfies the conditions (2.7) and (2.8).

Proof. The conditions (2.5) and (2.6) hold if and only if

$$
v(\mu(i), i)-p_{\mu(i)}=p_{i} \geq v(i, j)-p_{j} \quad(\forall(i, j) \in \bar{E}),
$$

which is equivalent to the combination of (2.7) and (2.8).

So far we consider characterizations of equilibria in the partnership formation problem. We then present necessary and sufficient conditions for the existence of an equilibrium by using the following dual pair of linear programming problems:

$$
\begin{array}{lll}
\text { (P) } & \text { Maximize } & \sum_{(i, j) \in E} v(i, j) x_{i j} \\
& \text { subject to } & \sum_{j \in N \backslash\{i\},(i, j) \in E} x_{i j} \leq 1 \quad(\forall i \in N), \\
& x_{i j} \geq 0 \quad(\forall(i, j) \in E), \\
\text { (D) } & \text { Minimize } & \sum_{i \in N} p_{i} \\
& \text { subject to } & p_{i}+p_{j} \geq v(i, j) \quad(\forall(i, j) \in E), \\
& p_{i} \geq 0 \quad(\forall i \in N) .
\end{array}
$$

Recall that $E$ is a set of distinct (unordered) pairs of agents in $N$. Problem (P) is a linear programming relaxation for the problem of finding a maximum-weight matching in $(N, E, v)$, where the weight of a matching $\mu: N \rightarrow N$ is given by $\sum_{i \in N} v(\mu(i), i)$. By the duality theorem for linear programming problems, the optimal value of $(\mathrm{P})$ is equal to the optimal value of $(\mathrm{D})$.

Proposition 2.2 (cf. Talman \& Yang (2011); Biró et al. (2012)). For the partnership formation problem $(N, E, v)$, the following three conditions are 
equivalent.

(a) There exists an equilibrium in $(N, E, v)$.

(b) Problem (P) has an integral optimal solution.

(c) The maximum weight of a matching in $(N, E, v)$ is equal to the optimal value of $(\mathrm{P})$ (or the optimal value of $(\mathrm{D})$ ).

From Proposition 2.2, we can obtain the following properties; the last property (iii) means that a matching and a payoff in an equilibrium can be chosen independently of each other.

Proposition 2.3 (cf. Talman \& Yang (2011)). Suppose that there exists an equilibrium in the partnership formation problem $(N, E, v)$. Let $\mu: N \rightarrow N$ be a matching and $p \in \mathbb{R}^{N}$ be a payoff.

(i) $\mu$ is an equilibrium matching if and only if it is a maximum-weight matching in $(N, E, v)$.

(ii) $p$ is an equilibrium payoff if and only if it is an optimal solution of the linear programming problem (D).

(iii) If $\mu$ is an equilibrium matching and $p$ is an equilibrium payoff, then $(\mu, p)$ is an equilibrium.

Example 1. To illustrate our results shown in this paper, we will use the following example of the partnership formation problem $(N, E, v)$ such that

$$
N=\{1,2,3,4,5\}, \quad E=\{(i, j) \mid i, j \in N, i \neq j\},
$$

and the values $v(i, j)$ for $(i, j) \in E$ are given by the following table:

\begin{tabular}{|c||c|c|c|c|c|}
\hline$i \backslash j$ & 1 & 2 & 3 & 4 & 5 \\
\hline \hline 1 & 0 & 5 & 4 & 1 & 2 \\
\hline 2 & 5 & 0 & 2 & 4 & 1 \\
\hline 3 & 4 & 2 & 0 & 3 & 1 \\
\hline 4 & 1 & 4 & 3 & 0 & 1 \\
\hline 5 & 2 & 1 & 1 & 1 & 0 \\
\hline
\end{tabular}

This problem has an equilibrium; indeed, the two matchings $\mu^{a}, \mu^{b}: N \rightarrow N$ given by

$$
\begin{aligned}
& \mu^{a}(1)=2, \mu^{a}(2)=1, \mu^{a}(3)=4, \mu^{a}(4)=3, \mu^{a}(5)=5, \\
& \mu^{b}(1)=3, \mu^{b}(2)=4, \mu^{b}(3)=1, \mu^{b}(4)=2, \mu^{b}(5)=5
\end{aligned}
$$


are equilibrium matchings. By Proposition 2.3 (i), they are maximum-weight matchings in $(N, E, v)$ with the weight equal to 8 . The set of equilibrium payoffs is given as

$$
\begin{aligned}
P=\left\{\left(p_{1}, p_{2}, p_{3}, p_{4}, p_{5}\right) \in \mathbb{R}^{N} \mid 2 \leq p_{1} \leq 3, p_{2}=5-p_{1},\right. \\
\left.p_{3}=4-p_{1}, p_{4}=-1+p_{1}, p_{5}=0\right\} .
\end{aligned}
$$

By Proposition 2.3 (iii), a pair $(\mu, p)$ of a matching $\mu: N \rightarrow N$ and a payoff $p \in \mathbb{R}^{N}$ is an equilibrium in $(N, E, v)$ if and only if $\mu \in\left\{\mu^{a}, \mu^{b}\right\}$ and $p \in P$.

Remark 2.4. Problem (P) is known as the fractional matching problem in the literature of combinatorial optimization (see, e.g., Schrijver (2003, Chapter 30)). The optimal value of problem (P) as well as an optimal solution can be computed by solving the maximum-weight matching problem on a bipartite graph (see, e.g., Nemhauser \& Trotter (1975), Pulleyblank (1987), Schrijver (2003, Chapter 30); see also Biró et al. (2012)).

The bipartite graph used for solving the problem $(\mathrm{P})$ is given as follows. Let $N^{\prime}$ be a copy of the set $N$, and denote by $i^{\prime} \in N^{\prime}$ the copy of $i \in N$, i.e., $N^{\prime}=\left\{i^{\prime} \mid i \in N\right\}$. Let us consider the bipartite graph $\left(N, N^{\prime} ; F_{0}\right)$ with vertex set $N \cup N^{\prime}$ and edge set $F_{0}$ given by

$$
F_{0}=\left\{\left(i, j^{\prime}\right) \in N \times N^{\prime} \mid(i, j) \in E\right\} .
$$

Therefore, for each $(i, j) \in E$ with $i \neq j$, set $F_{0}$ contains two edges $\left(i, j^{\prime}\right)$ and $\left(j, i^{\prime}\right)$, and for each $i \in N$, there is no edge between the vertices $i \in N$ and $i^{\prime} \in N^{\prime}$. For $\left(i, j^{\prime}\right) \in F_{0}$, we define the edge weight $w\left(i, j^{\prime}\right)$ by $w\left(i, j^{\prime}\right)=v(i, j)$.

Then, the half of the weight of a maximum-weight matching in the bipartite graph $\left(N, N^{\prime} ; F_{0}\right)$ is equal to the optimal value of the problem $(\mathrm{P})$. Moreover, for a maximum-weight matching $M \subseteq F_{0}$ in $\left(N, N^{\prime} ; F_{0}\right)$, the vector $x^{*}=\left(x_{i j}^{*}\right)$ $(i, j) \in E)$ given by

$$
x_{i j}^{*}=\left\{\begin{array}{cl}
1 & \left(\text { if } M \text { contains both of }\left(i, j^{\prime}\right) \text { and }\left(j, i^{\prime}\right)\right) \\
1 / 2 & \left(\text { if } M \text { contains exactly one of }\left(i, j^{\prime}\right) \text { and }\left(j, i^{\prime}\right)\right), \\
0 & \text { (otherwise, i.e., } \left.M \text { contains neither of }\left(i, j^{\prime}\right) \text { and }\left(j, i^{\prime}\right)\right)
\end{array}\right.
$$

is an optimal solution of $(\mathrm{P})$.

In the following sections we will use a bipartite graph similar to $\left(N, N^{\prime} ; F_{0}\right)$ to reveal the connection between the partnership formation problem and the assignment game. 


\subsection{Assignment Game}

The assignment game is a special case of the partnership formation problem, where the set of agents $N$ is partitioned into two disjoint sets $A$ and $B$ corresponding to sellers and buyers, respectively, and any two distinct sellers (buyers) cannot be a pair.

In the following, we represent an instance of the assignment game by a tuple $(A, B, F, w)$, where $(A, B ; F)$ is a bipartite graph and $w: F \rightarrow \mathbb{R}_{+}$is an edge weight function taking non-negative real numbers. The vertex sets $A$ and $B$ represent the sets of sellers and buyers, respectively, and $F \subseteq A \times B$ is the set of possible pairs of sellers and buyers, i.e., buyer $i$ and seller $j$ can be a pair if and only if $(i, j) \in F$. For $(i, j) \in F, w(i, j)$ represents the joint values generated by pairs of agents $i \in A$ and $j \in B$. Note that the original model of the assignment game coincides with the case with $F=A \times B=\{(i, j) \mid i \in A, j \in B\}$, i.e., any seller and buyer can be a pair.

For notational convenience, we consider a dummy seller, denote by 0 , and regard each buyer $j \in B$ with no partner as a pair $(0, j)$ with the dummy seller. We assume that the joint value generated by a pair with the dummy seller is zero (i.e., $w(0, j)=0$ for all $j \in B$ ), and the dummy seller can be a pair with an arbitrary number of buyers. A matching in the assignment game $(A, B, F, w)$ is a function $\eta: B \rightarrow A \cup\{0\}$ satisfying the following conditions:

$(\eta(j), j) \in F$ for every $j \in B$ with $\eta(j) \neq 0$

(i.e., every pair $(\eta(j), j)$ is "feasible"),

for each $i \in A$ there exists at most one $j \in B$ with $\eta(j)=i$

(i.e., every seller can be a pair with at most one buyer).

Vectors $q=\left(q_{i} \mid i \in A\right) \in \mathbb{R}^{A}$ and $r=\left(r_{j} \mid j \in B\right) \in \mathbb{R}^{B}$ are called sellers' payoff and buyers' payoff, respectively. A seller's payoff is sometimes called a price vector. The pair $(q, r)$ is simply called a payoff.

The concept of equilibrium in the partnership formation problem is specialized to the assignment game as follows. The tuple $(\eta, q, r)$ of a matching $\eta$ and a payoff $(q, r)$ is called an equilibrium in the assignment game $(A, B, F, w)$ 
if the following conditions hold with $q_{0}=0$ :

$$
\begin{aligned}
& q_{i}+r_{j} \geq w(i, j) \quad(\forall(i, j) \in F), \\
& q_{\eta(j)}+r_{j}=w(\eta(j), j) \quad(\forall j \in B), \\
& q_{i} \geq 0 \quad(\forall i \in A), \quad r_{j} \geq 0 \quad(\forall j \in B), \\
& q_{i}=0 \quad(\forall i \in A \backslash\{\eta(j) \mid j \in B\}) .
\end{aligned}
$$

Every assignment game has an equilibrium (Shapley \& Shubik, 1971).

The conditions (2.12) and (2.13) can be rewritten in terms of demand correspondences. For a vector of sellers' payoff $q \in \mathbb{R}^{A}$ and a buyer $j \in B$, we define the demand correspondence $\widetilde{D}_{j}(q) \subseteq A \cup\{0\}$ of buyer $j$ by

$$
\widetilde{D}_{j}(q)=\arg \max \left\{w(i, j)-q_{i} \mid i \in A \cup\{0\},(i, j) \in F \cup\{(0, j)\}\right\} .
$$

We consider the following conditions:

$$
\begin{aligned}
& \eta(j) \in \widetilde{D}_{j}(q) \quad(\forall j \in B), \\
& r_{j}=\max \left\{w(i, j)-q_{i} \mid i \in A \cup\{0\},(i, j) \in F \cup\{(0, j)\}\right\} \quad(\forall j \in B),
\end{aligned}
$$

where $q_{0}=0$.

Proposition 2.5. Let $\eta: B \rightarrow A \cup\{0\}$ be a matching in the assignment game $(A, B, F, w)$, and $(q, r) \in \mathbb{R}^{A} \times \mathbb{R}^{B}$ be a payoff. Then, the tuple $(\eta, q, r)$ is an equilibrium if and only if it satisfies the conditions (2.14), (2.15), (2.17), and (2.18).

A matching $\eta$ and a payoff $(q, r)$ in an equilibrium $(\eta, q, r)$ are called an equilibrium matching and an equilibrium payoff, respectively. Equilibrium matching and payoff can be characterized as optimal solutions of certain optimization problems (see, e.g., Shapley \& Shubik (1971); Roth \& Sotomayor (1990)). In addition, any combination of an equilibrium matching and an equilibrium payoff gives an equilibrium.

Proposition 2.6. Let $\eta: B \rightarrow A \cup\{0\}$ be a matching in the assignment game $(A, B, F, w)$, and $(q, r) \in \mathbb{R}^{A} \times \mathbb{R}^{B}$ be a payoff.

(i) $\eta$ is an equilibrium matching if and only if it maximizes the weight $\sum_{j \in B} w(\eta(j), j)$ among all matchings. 
(ii) $(q, r)$ is an equilibrium payoff if and only if it is an optimal solution of the following linear programming problem:

$$
\begin{array}{cl}
\text { Minimize } & \sum_{i \in A} q_{i}+\sum_{j \in B} r_{j} \\
\text { subject to } & q_{i}+r_{j} \geq w(i, j) \quad(\forall(i, j) \in F), \\
& q_{i} \geq 0 \quad(\forall i \in A), \quad r_{j} \geq 0 \quad(\forall j \in B) .
\end{array}
$$

(iii) If $\eta$ is an equilibrium matching and $(q, r)$ is an equilibrium payoff, then $(\eta, q, r)$ is an equilibrium.

Define

$$
\begin{aligned}
& \mathscr{H}_{0}=\left\{q \in \mathbb{R}^{A} \mid(q, r)\right. \text { is an equilibrium payoff } \\
& \left.\qquad \text { in }(A, B, F, w) \text { for some } r \in \mathbb{R}^{B}\right\} .
\end{aligned}
$$

If we regard $q \in \mathbb{R}^{A}$ as a price vector of sellers' goods, then $\mathscr{H}_{0}$ can be seen as the set of equilibrium price vectors in the assignment game. Hence, a minimal vector in the set $\mathscr{H}_{0}$ is uniquely determined (see, e.g., Shapley \& Shubik (1971)).

It is known that if an equilibrium price vector $q \in \mathscr{H}_{0}$ is fixed, then the corresponding buyers' payoff $r$ in an equilibrium payoff $(q, r)$ is uniquely determined by (2.18).

Proposition 2.7. Let $(q, r) \in \mathbb{R}^{A} \times \mathbb{R}^{B}$ be a payoff in the assignment game $(A, B, F, w)$. Then, $(q, r)$ is an equilibrium payoff if and only if $q \in \mathscr{H}_{0}$ and $r$ is given by (2.18).

An equilibrium matching can be characterized by using demand correspondences.

Proposition 2.8. Let $\eta: B \rightarrow A \cup\{0\}$ be a matching in the assignment game $(A, B, F, w)$, and $q \in \mathbb{R}^{A}$ be a vector with $q \in \mathscr{H}_{0}$. Then, $\eta$ is an equilibrium matching if and only if $\eta$ and $q$ satisfy (2.15) and (2.17).

Proof. We define a vector $r \in \mathbb{R}^{B}$ by (2.18). By Proposition 2.7, $(q, r)$ is an equilibrium payoff, and therefore it satisfies the condition (2.14). This fact, together with Proposition 2.6 (iii), implies that $\eta$ is an equilibrium matching if and only if $(\eta, q, r)$ is an equilibrium. By Proposition $2.5,(\eta, q, r)$ is an equilibrium if and only if $\eta$ is a matching satisfying (2.15) and (2.17). 
Finally, we present a price adjustment process (also known as a dynamic auction) for computation of a vector in $\mathscr{H}_{0}$. It is known that the unique minimal vector in $\mathscr{H}_{0}$ can be obtained by an ascending-type price adjustment process, which is explained below.

For a vector $q \in \mathbb{R}_{+}^{A}$ and a set $Y \subseteq A$, we define

$$
\begin{aligned}
\widetilde{O}(Y, q) & =\left\{j \in B \mid \widetilde{D}_{j}(q) \subseteq Y\right\}, \\
\widetilde{U}(Y, q) & =\left\{j \in B \mid \widetilde{D}_{j}(q) \cap Y \neq \emptyset\right\} .
\end{aligned}
$$

The set $\widetilde{O}(Y, q)$ consists of buyers who only demand sellers in $Y$ at price $q$, while $\widetilde{U}(Y, q)$ is the set of buyers who demand some seller in $Y$ at price $q$. Obviously, $\widetilde{O}(Y, q) \subseteq \widetilde{U}(Y, q)$ holds. A set $Y \subseteq A$ is said to be overdemanded if $|\widetilde{O}(Y, q)|>|Y|$. A set $X \subseteq A$ is said to be in excess demand at price $q$ if it satisfies

$$
|\widetilde{U}(Y, q) \cap \widetilde{O}(X, q)|>|Y| \quad(\emptyset \neq \forall Y \subseteq X) .
$$

It is known that a maximal set in excess demand is uniquely determined (Mo et al. (1988); Sankaran (1994)). Moreover, the maximal set $X$ in excess demand is an overdemanded set that maximizes the value $|\widetilde{O}(X, q)|-|X|$ (Murota et al. (2016)).

An ascending-type price adjustment process due to Mo et al. (1988) and Sankaran (1994), which is a variant of the one in Demange et al. (1986), is described as follows. We here assume that the values $w(i, j)((i, j) \in F)$ are non-negative integers; this assumption implies that the minimal vector in $\mathscr{H}_{0}$ is integral.

\section{Algorithm VICKREYENGLISH}

Step 0: Set $q \in \mathbb{Z}^{A}$ by $q:=(0,0, \ldots, 0)$.

Step 1: Collect the demand correspondences $\widetilde{D}_{j}(q)$ for $j \in B$.

Step 2: If $|\widetilde{O}(S, q)| \leq|S|$ holds for every $S \subseteq A$, then output $q$ and stop.

Step 3: Find the unique maximal set $\widetilde{S}^{*} \subseteq A$ in excess demand at payoff $q$, update $q$ by $q_{i}:=q_{i}+1\left(i \in \widetilde{S}^{*}\right)$, and go to Step 1 .

Proposition 2.9. For the assignment game $(A, B, F, w)$, assume that the values $w(i, j)((i, j) \in F)$ are non-negative integers. Then, the algorithm VICKREYENGLISH outputs the unique minimal vector in $\mathscr{H}_{0}$ (i.e., the unique minimal equilibrium price vector). 
For more price adjustment processes for computing an equilibrium price vector, see Appendix and also Andersson et al. (2010), Andersson \& Erlanson (2013) and Mishra \& Parkes (2009).

\section{REDUCTION OF PARTNERSHIP FORMATION PROBLEM TO ASSIGNMENT GAME}

As mentioned in Section 2.2, the assignment game is a special case of the partnership formation problem. Hence, if we can compute an equilibrium in the partnership formation problem, then we can also compute an equilibrium in the assignment game.

In this section, we show that the converse is also true in some sense, i.e., if we can compute an equilibrium in the assignment game, then we can also compute an equilibrium in the partnership formation problem if it exists. For this, we associate an assignment game with the partnership formation problem in Section 3.1, and present its fundamental properties in Section 3.2. In Section 3.3 we present the main theorems on the relationship between the partnership formation problem and the associated assignment game. Based on these theorems, we propose an algorithm for computing an equilibrium in the partnership formation problem. Proofs are given in Section 3.4.

\subsection{Assignment Game Associated with Partnership Formation Problem}

For the partnership formation problem $(N, E, v)$, we associate the assignment game $\left(N, N^{\prime}, F, w\right)$ as follows, where $F_{0}$ is given by (2.11).

$$
\begin{aligned}
N^{\prime} & =\left\{i^{\prime} \mid i \in N\right\}, \text { where } i^{\prime} \text { is a copy of } i, \\
F & =F_{0} \cup\left\{\left(i, i^{\prime}\right) \mid i \in N\right\} \\
& =\left\{\left(i, j^{\prime}\right) \in N \times N^{\prime} \mid(i, j) \in E\right\} \cup\left\{\left(i, i^{\prime}\right) \mid i \in N\right\}, \\
w\left(i, j^{\prime}\right) & =\left\{\begin{array}{ll}
v(i, j) & \text { if } i \neq j), \\
0 & (\text { if } i=j) .
\end{array} \quad\left(\left(i, j^{\prime}\right) \in F\right) .\right.
\end{aligned}
$$

We see that the associated assignment game $\left(N, N^{\prime}, F, w\right)$ is similar to the bipartite graph $\left(N, N^{\prime} ; F_{0}\right)$ in Remark 2.4.

Example 2. To illustrate the associated assignment game defined above, we consider the partnership formation problem $(N, E, v)$ in Example 1. The 
associated assignment game $\left(N, N^{\prime}, F, w\right)$ is given by

$$
\begin{aligned}
& N=\{1,2,3,4,5\}, \quad N^{\prime}=\left\{1^{\prime}, 2^{\prime}, 3^{\prime}, 4^{\prime}, 5^{\prime}\right\}, \\
& F=\left\{\left(i, j^{\prime}\right) \mid i \in N, j^{\prime} \in N^{\prime}\right\},
\end{aligned}
$$

and values $w\left(i, j^{\prime}\right)$ for $\left(i, j^{\prime}\right) \in F$ are given by the following table:

\begin{tabular}{|c||c|c|c|c|c|}
\hline$i \backslash j^{\prime}$ & $1^{\prime}$ & $2^{\prime}$ & $3^{\prime}$ & $4^{\prime}$ & $5^{\prime}$ \\
\hline \hline 1 & 0 & 5 & 4 & 1 & 2 \\
\hline 2 & 5 & 0 & 2 & 4 & 1 \\
\hline 3 & 4 & 2 & 0 & 3 & 1 \\
\hline 4 & 1 & 4 & 3 & 0 & 1 \\
\hline 5 & 2 & 1 & 1 & 1 & 0 \\
\hline
\end{tabular}

The assignment game $\left(N, N^{\prime}, F, w\right)$ has two equilibrium matchings $\eta^{a}, \eta^{b}$ : $N^{\prime} \rightarrow N$ given as

$$
\begin{aligned}
& \eta^{a}\left(1^{\prime}\right)=2, \eta^{a}\left(2^{\prime}\right)=1, \eta^{a}\left(3^{\prime}\right)=4, \eta^{a}\left(4^{\prime}\right)=3, \eta^{a}\left(5^{\prime}\right)=5 \\
& \eta^{b}\left(1^{\prime}\right)=3, \eta^{b}\left(2^{\prime}\right)=4, \eta^{b}\left(3^{\prime}\right)=1, \eta^{b}\left(4^{\prime}\right)=2, \eta^{b}\left(5^{\prime}\right)=5
\end{aligned}
$$

The set of equilibrium payoffs is given as

$$
\begin{aligned}
\widetilde{P}=\left\{(q, r) \in \mathbb{R}^{N} \times \mathbb{R}^{N^{\prime}} \mid\right. & 2 \leq q_{1} \leq 3,2 \leq q_{2} \leq 3, \\
& q_{3}=q_{2}-1, q_{4}=q_{1}-1, q_{5}=0, \\
& r_{1^{\prime}}=5-q_{2}, r_{2^{\prime}}=5-q_{1}, r_{3^{\prime}}=3-q_{4}, \\
& \left.r_{4^{\prime}}=3-q_{3}, r_{5^{\prime}}=0\right\} .
\end{aligned}
$$

By Proposition 2.6 (iii), a tuple $(\eta, q, r)$ is an equilibrium in the assignment game $\left(N, N^{\prime}, F, w\right)$ if and only if $\eta \in\left\{\eta^{a}, \eta^{b}\right\}$ and $(q, r) \in \widetilde{P}$.

Assignment games associated with the partnership formation problem are also considered in Biró et al. (2012) and Chiappori et al. (2014), which are similar to ours, but different in the use of pairs $\left\{\left(i, i^{\prime}\right) \mid i \in N\right\}$. In the following, we discuss the difference among the three assignment games. While the difference is subtle, it is crucial to our main results.

The associated assignment game in Biró et al. (2012) is obtained from ours by deleting the pairs $\left\{\left(i, i^{\prime}\right) \mid i \in N\right\}$ from $F$, i.e., the set $F$ is replaced with $F_{0}$ given by (2.11). Hence, the assignment game in Biró et al. (2012) 
is given by the bipartite graph $\left(N, N^{\prime} ; F_{0}\right)$ in Remark 2.4. Biró et al. (2012) use the maximum weight of a matching in the associated assignment game to characterize the existence of an equilibrium in the partnership formation problem $(N, E, v)$ (see Proposition 2.2 and Remark 2.4).

The associated assignment game in Chiappori et al. (2014) uses the pairs $\left\{\left(i, i^{\prime}\right) \mid i \in N\right\}$ as in ours, but for a different purpose. Chiappori et al. (2014) consider a more general model of the partnership formation problem, where each $i \in N$ corresponds to a type of agents and there are multiple agents of the same type. Each pair $\left(i, i^{\prime}\right)$ in the assignment game of Chiappori et al. (2014) is used to represent a pair of agents of the type $i \in N$. In contrast, in our assignment game, each pair $\left(i, i^{\prime}\right)$ corresponds to a single agent $i \in N$ with no partner. Chiappori et al. (2014) use the associated assignment game to provide a necessary and sufficient condition for the existence of an equilibrium in their general model, in a similar way as in Biró et al. (2012).

\subsection{Properties of Equilibria in the Associated Assignment Game}

The associated assignment game $\left(N, N^{\prime}, F, w\right)$ defined in Section 3.1 is "symmetric" in the sense that $\left(i, j^{\prime}\right) \in F$ holds if and only if $\left(j, i^{\prime}\right) \in F$, and the pairs $\left(i, j^{\prime}\right)$ and $\left(j, i^{\prime}\right)$ have the same weight. Due to the symmetric structure, various nice properties of equilibria in $\left(N, N^{\prime}, F, w\right)$ can be obtained. In the following discussion, we often identify the set $N^{\prime}$ with $N$ through the natural one-to-one correspondence, and regard a vector in $\mathbb{R}^{N^{\prime}}$ (resp., $\mathbb{R}^{N}$ ) as a vector in $\mathbb{R}^{N}$ (resp., $\mathbb{R}^{N^{\prime}}$.

We consider a matching in $\left(N, N^{\prime}, F, w\right)$ such that every agent in $N^{\prime} \cup N$ has a partner. We call such a matching a perfect matching, following the graph theory terminology. Note that a perfect matching indeed exists in $\left(N, N^{\prime}, F, w\right)$ since $\left\{\left(i, i^{\prime}\right) \mid i \in N\right\} \subseteq F$.

The next proposition shows that the assignment game $\left(N, N^{\prime}, F, w\right)$ has an equilibrium matching that is a perfect matching. It should be noted that this property does not hold for general assignment games.

Proposition 3.1. There exists an equilibrium matching $\eta: N^{\prime} \rightarrow N \cup\{0\}$ in the assignment game $\left(N, N^{\prime}, F, w\right)$ such that $\eta$ is a perfect matching.

Proof. Proof is given in Section 3.4.1.

In the following discussion, we consider a perfect matching whenever we refer to a matching in $\left(N, N^{\prime}, F, w\right)$. Use of a perfect matching is a key to obtain 
the main results of this paper.

For the assignment game $\left(N, N^{\prime}, F, w\right)$ and a sellers' payoff $q \in \mathbb{R}^{N}$, we have defined the demand correspondence $\widetilde{D}_{j^{\prime}}(q) \subseteq N \cup\{0\}$ of buyer $j^{\prime} \in N^{\prime}$ by (2.16), i.e.,

$$
\widetilde{D}_{j^{\prime}}(q)=\arg \max \left\{w\left(i, j^{\prime}\right)-q_{i} \mid i \in N \cup\{0\},\left(i, j^{\prime}\right) \in F \cup\left\{\left(0, j^{\prime}\right)\right\}\right\} .
$$

By the definition of the weight $w\left(i, j^{\prime}\right)$, we have

$$
\widetilde{D}_{j^{\prime}}(q)=\arg \max \left\{v(i, j)-q_{i} \mid i \in N \cup\{0\},(i, j) \in E \cup\{(0, j)\}\right\}
$$

with $v(0, j)=0$, and therefore $\widetilde{D}_{j^{\prime}}(q) \backslash\{0\}=D_{j}(q)$ holds if $\widetilde{D}_{j^{\prime}}(q) \backslash\{0\} \neq \emptyset$ (i.e., $\left.\max \left\{w\left(i, j^{\prime}\right)-q_{i} \mid i \in N,\left(i, j^{\prime}\right) \in F\right\} \geq 0\right)$.

For the assignment game $\left(N, N^{\prime}, F, w\right)$, we have defined the set $\mathscr{H}_{0} \subseteq \mathbb{R}^{N}$ of equilibrium price vectors by (2.19), i.e., $\mathscr{H}_{0}$ is given as

$$
\begin{aligned}
& \mathscr{H}_{0}=\left\{q \in \mathbb{R}^{N} \mid(q, r)\right. \text { is an equilibrium payoff } \\
& \left.\qquad \text { in }\left(N, N^{\prime}, F, w\right) \text { for some } r \in \mathbb{R}^{N^{\prime}}\right\} .
\end{aligned}
$$

We show that the definition and characterizations (Propositions 2.7 and 2.8) of an equilibrium $(\eta, q, r)$ in the associated assignment game $\left(N, N^{\prime}, F, w\right)$ can be simplified if we restrict $\eta$ to a perfect matching. Recall that an equilibrium in the assignment game is defined by the four conditions (2.12)-(2.15).

Lemma 3.2. Let $\eta: N^{\prime} \rightarrow N$ and $(q, r) \in \mathbb{R}^{N} \times \mathbb{R}^{N^{\prime}}$ be a perfect matching and a payoff in the assignment game $\left(N, N^{\prime}, F, w\right)$, respectively.

(i) $(\eta, q, r)$ is an equilibrium in $\left(N, N^{\prime}, F, w\right)$ if and only if the following conditions hold:

$$
\begin{aligned}
& q_{i}+r_{j^{\prime}} \geq w\left(i, j^{\prime}\right) \quad\left(\forall\left(i, j^{\prime}\right) \in F\right), \\
& q_{\eta\left(j^{\prime}\right)+r_{j^{\prime}}=w\left(\eta\left(j^{\prime}\right), j^{\prime}\right)} \quad\left(\forall j^{\prime} \in N^{\prime}\right), \\
& q_{i} \geq 0 \quad(\forall i \in N), \quad r_{j^{\prime}} \geq 0 \quad\left(\forall j^{\prime} \in N^{\prime}\right) .
\end{aligned}
$$

(ii) $(q, r)$ is an equilibrium payoff in $\left(N, N^{\prime}, F, w\right)$ if and only if $q \in \mathscr{H}_{0}$ and $r$ is given by

$$
r_{j^{\prime}}=\max \left\{w\left(i, j^{\prime}\right)-q_{i} \mid i \in N,\left(i, j^{\prime}\right) \in F\right\} \quad\left(j^{\prime} \in N^{\prime}\right) .
$$


Hence, the formula (3.5) can be rewritten as

$$
\begin{array}{r}
\mathscr{H}_{0}=\left\{q \in \mathbb{R}^{N} \mid \text { the pair }(q, r) \text { of } q \text { and } r \in \mathbb{R}^{N^{\prime}} \text { given by }(3.9)\right. \\
\text { is an equilibrium payoff in } \left.\left(N, N^{\prime}, F, w\right)\right\} .
\end{array}
$$

(iii) Suppose that $q \in \mathscr{H}_{0}$ holds. Then, we have $\widetilde{D}_{j}(q) \backslash\{0\} \neq \emptyset(\forall j \in N)$. Moreover, $\eta$ is an equilibrium matching in $\left(N, N^{\prime}, F, w\right)$ if and only if

$$
\eta\left(j^{\prime}\right) \in \widetilde{D}_{j^{\prime}}(q) \backslash\{0\} \quad\left(\forall j^{\prime} \in N^{\prime}\right) .
$$

Proof. Proof is given in Section 3.4.2.

\subsection{Theorem and Algorithm for Partnership Formation Problem}

We first present a characterization of equilibria in the partnership formation problem $(N, E, v)$ in terms of the associated assignment game $\left(N, N^{\prime}, F, w\right)$. For a matching $\mu: N \rightarrow N$ in $(N, E, v)$, we define a matching $\eta_{\mu}: N^{\prime} \rightarrow N$ in $\left(N, N^{\prime}, F, w\right)$ associated with $\mu$ by

$$
\eta_{\mu}\left(j^{\prime}\right)=\mu(j) \quad\left(j^{\prime} \in N^{\prime}\right) .
$$

Note that $\eta_{\mu}$ is a perfect matching in $\left(N, N^{\prime}, F, w\right)$.

Theorem 3.3. Let $\mu: N \rightarrow N$ and $p \in \mathbb{R}^{N}$ be a matching and a payoff in the partnership formation problem $(N, E, v)$, respectively. Also, let $\eta_{\mu}: N^{\prime} \rightarrow N$ be the (perfect) matching in the associated assignment game $\left(N, N^{\prime}, F, w\right)$ given by (3.11). Then, the following three statements are equivalent:

(a) $(\mu, p)$ is an equilibrium in $(N, E, v)$.

(b) $\left(\eta_{\mu}, p, p\right)$ is an equilibrium in $\left(N, N^{\prime}, F, w\right)$.

(c) $\left(\eta_{\mu}, q, r\right)$ is an equilibrium in $\left(N, N^{\prime}, F, w\right)$ for some payoff $(q, r) \in \mathbb{R}^{N} \times$ $\mathbb{R}^{N^{\prime}}$ with $(1 / 2)(q+r)=p$.

Proof. Proof is given in Section 3.4.3.

The next theorem clarifies the relationship between equilibrium matchings (resp., payoffs) in the partnership formation problem $(N, E, v)$ and equilibrium matchings (resp., payoffs) in the associated assignment game $\left(N, N^{\prime}, F, w\right)$. 


\section{Theorem 3.4.}

(i) For a matching $\mu: N \rightarrow N$ in the partnership formation problem $(N, E, v)$, let $\eta_{\mu}: N^{\prime} \rightarrow N$ be the (perfect) matching given by (3.11) in the associated assignment game $\left(N, N^{\prime}, F, w\right)$. Then, $\mu$ is an equilibrium matching in $(N, E, v)$ if and only if $\eta_{\mu}$ is an equilibrium matching in $\left(N, N^{\prime}, F, w\right)$.

(ii) Suppose that there exists an equilibrium in $(N, E, v)$. Then, a payoff $p \in \mathbb{R}^{N}$ is an equilibrium payoff in $(N, E, v)$ if and only if there exists an equilibrium payoff $(q, r) \in \mathbb{R}^{N} \times \mathbb{R}^{N^{\prime}}$ in $\left(N, N^{\prime}, F, w\right)$ such that $(1 / 2)(q+r)=p$.

Proof. Proof is given in Section 3.4.4.

We finally show that an equilibrium in the partnership formation problem $(N, E, v)$ can be obtained by using an equilibrium payoff in the associated assignment game $\left(N, N^{\prime}, F, w\right)$, provided that an equilibrium exists in $(N, E, v)$.

Theorem 3.5. Let $\mu: N \rightarrow N$ be a matching in the partnership formation problem $(N, E, v)$, and $q \in \mathbb{R}^{N}$ be a payoff with $q \in \mathscr{H}_{0}$.

(i) $\mu$ is an equilibrium matching in $(N, E, v)$ if and only if

$$
\mu(i) \in D_{i}(q) \quad(\forall i \in N) .
$$

(ii) Suppose that $\mu$ is an equilibrium matching in $(N, E, v)$. Define $p \in \mathbb{R}^{N}$ by

$$
p_{i}=(1 / 2)\left(v(\mu(i), i)+q_{i}-q_{\mu(i)}\right) \quad(i \in N) .
$$

Then, $p$ is an equilibrium payoff in $(N, E, v)$.

Proof. Proof is given in Section 3.4.5.

Example 3. To illustrate the statements of Theorem 3.5, we consider the partnership formation problem $(N, E, v)$ in Example 1 . We have

$$
\begin{aligned}
\mathscr{H}_{0}=\left\{q \in \mathbb{R}^{N} \mid 2 \leq q_{1} \leq 3,2\right. & \leq q_{2} \leq 3, \\
& \left.q_{3}=q_{2}-1, q_{4}=q_{1}-1, q_{5}=0\right\} .
\end{aligned}
$$

For every $q \in \mathscr{H}_{0}$, it holds that

$$
\begin{aligned}
& \{2,3\} \subseteq D_{1}(q)=D_{4}(q) \subseteq\{2,3,5\}, \\
& D_{2}(q)=\{1,4\} \\
& \{1,4\} \subseteq D_{3}(q) \subseteq\{1,4,5\} \\
& \{5\} \subseteq D_{5}(q) \subseteq\{1,3,4,5\} .
\end{aligned}
$$


It is observed that the (equilibrium) matchings $\mu^{a}, \mu^{b}: N \rightarrow N$ given by (2.9) and (2.10), respectively, satisfy the condition $\mu^{a}(j), \mu^{b}(j) \in D_{j}(q)(\forall j \in N)$ by Theorem 3.5 (i).

For the matching $\mu=\mu_{a}$ and the payoff $q=(2,3,2,1,0) \in \mathscr{H}_{0}$, the vector $p \in \mathbb{R}^{N}$ given by (3.13) is equal to $(2,3,2,1,0)$, which is an equilibrium payoff in $(N, E, v)$ by Theorem 3.5 (ii).

Theorem 3.5 implies that an equilibrium in the partnership formation problem $(N, E, v)$ can be found by the following algorithm. The idea of the algorithm is as follows. Let $q \in \mathbb{R}^{N}$ be a vector in $\mathscr{H}_{0}$, which can be computed by finding an equilibrium in the associated assignment game $\left(N, N^{\prime}, F, w\right)$ (see Remark 3.6). By Theorem 3.5 (i), there exists an equilibrium matching in $(N, E, v)$ if and only if there exists a matching $\mu: N \rightarrow N$ in $(N, E, v)$ satisfying the condition (3.12). Therefore, it suffices to check the existence of a matching satisfying the condition (3.12), which can be done by finding a maximumcardinality matching on a certain undirected graph (see Remark 3.7).

\section{Algorithm ComputeEQuilibrium}

Step 1: Find a vector $q \in \mathscr{H}_{0}$.

Step 2: If there exists no matching $\mu: N \rightarrow N$ in $(N, E, v)$ satisfying (3.12), then assert that "no equilibrium exists in $(N, E, v)$ " and stop.

Step 3: Find a matching $\mu: N \rightarrow N$ in $(N, E, v)$ satisfying (3.12), and let $p \in \mathbb{R}^{N}$ be a vector given by (3.13).

Output $(\mu, p)$ as an equilibrium of $(N, E, v)$.

Remark 3.6. We discuss the computation of a vector $q \in \mathscr{H}_{0}$ in Step 1. If the information of the demand correspondences $\widetilde{D}_{j^{\prime}}(q)\left(j^{\prime} \in N^{\prime}\right)$ is available, then a vector $q \in \mathscr{H}_{0}$ can be computed by the algorithm VICKREYENGLISH in Section 2.2 or other price adjustment processes (see Appendix). In the case where the information of the sets $D_{j}(q)(j \in N)$ is available, a vector $q \in \mathscr{H}_{0}$ can be computed by an adjustment process by Andersson et al. (2014b); see Section 4 for details.

Remark 3.7. Computation of a matching $\mu$ in $(N, E, v)$ satisfying (3.12) can be reduced to finding a maximum-cardinality matching in an undirected graph with vertex set $N$ and the edge set given by $\left\{(i, j) \mid i, j \in N, i \in D_{j}(p)\right\}$ (see Andersson et al. (2014b)). A maximum-cardinality matching in an undirected graph can be found in strongly polynomial time, i.e., the time required to find 
a maximum-cardinality matching can be bounded by a polynomial in $n=|N|$ (see, e.g., Schrijver (2003)).

Remark 3.8. The algorithm COMPUTEEQUILIBRIUM requires the information about the joint values of agents pairs only in the computation of an equilibrium payoff $p$ in Step 3, and other steps can be performed by using demand correspondences only. This means that the information about the joint values of agents pairs is not needed when we just check the existence of an equilibrium and find an equilibrium matching (if it exists).

\subsection{Proofs}

\subsubsection{Proof of Proposition 3.1}

We prove the claim by a graph-theoretic argument. Let us consider a bipartite graph $G=\left(N, N^{\prime} ; F\right)$ with vertex set $N \cup N^{\prime}$, edge set $F$, and edge weight $w\left(i, j^{\prime}\right)$ for $\left(i, j^{\prime}\right) \in F$. Recall that a matching in the graph $G$ is a set $M \subseteq F$ of edges such that for each vertex $i \in N \cup N^{\prime}$ there exists at most one edge in $M$ incident to $i$. It is easy to see that matchings in the assignment game $\left(N, N^{\prime}, F, w\right)$ have a natural one-to-one correspondence with matchings in $G$; moreover, Proposition 2.6 (i) implies that a matching in $\left(N, N^{\prime}, F, w\right)$ is an equilibrium matching if and only if its corresponding matching in $G$ is a maximum-weight matching. Hence, to prove Proposition 3.1 it suffices to show that there exists a maximum-weight matching in $G$ that is a perfect matching.

Let $M \subseteq F$ be a maximum-weight matching in $G$. We assume that $M$ is not a perfect matching. Due to the symmetry of the graph $G$,

$$
M^{\prime}=\left\{\left(j, i^{\prime}\right) \in F \mid\left(i, j^{\prime}\right) \in M\right\}
$$

is also a maximum-weight matching in $G$.

Using the two matchings $M$ and $M^{\prime}$, we define an edge set $X$ as follows. For a vertex $i$ in the graph $G$, we denote by $\operatorname{deg}_{X}(i)$ the number of edges in $X$ incident to $i$. We initially set $X=M \cup M^{\prime}$. Then, we have $\operatorname{deg}_{X}(i)=$ $\operatorname{deg}_{X}\left(i^{\prime}\right) \leq 2$ for $i \in N$. For each $i \in N$, if $\operatorname{deg}_{X}(i)=\operatorname{deg}_{X}\left(i^{\prime}\right)=1$ then we add to $X$ (one copy of) the edge $\left(i, i^{\prime}\right)$, and if $\operatorname{deg}_{X}(i)=\operatorname{deg}_{X}\left(i^{\prime}\right)=0$ then we add to $X$ two copies of the edge $\left(i, i^{\prime}\right)$. Then, the resulting edge set $X$ satisfies $\operatorname{deg}_{X}(i)=\operatorname{deg}_{X}\left(i^{\prime}\right)=2$ for each $i \in N$. This implies that $X$ can be decomposed into two perfect matchings, which are denoted as $X_{1}$ and $X_{2}$. Moreover, the total 
weight of the edge set $X$ is twice the weight of a maximum-weight matching since $X$ contains all edges in $M \cup M^{\prime}$ and each edge $\left(i, i^{\prime}\right)$ has zero weight. Hence, both of the matchings $X_{1}$ and $X_{2}$ are maximum-weight matchings that are perfect matchings.

\subsubsection{Proof of Lemma 3.2}

[Proof of (i)] By definition, the tuple $(\eta, q, r)$ is an equilibrium if and only if it satisfies the conditions (3.6)-(3.8) and an additional condition that $q_{i}=0$ holds for all $i \in N \backslash\left\{\eta\left(j^{\prime}\right) \mid j^{\prime} \in N^{\prime}\right\}$. Since $\eta$ is a perfect matching, the set $N \backslash\left\{\eta\left(j^{\prime}\right) \mid j^{\prime} \in N^{\prime}\right\}$ is empty, and therefore the additional condition holds immediately. Hence, the claim (i) holds.

[Proof of (ii)] By Proposition 2.7, $(q, r)$ is an equilibrium payoff in $\left(N, N^{\prime}, F, w\right)$ if and only if $q \in \mathscr{H}_{0}$ and $r$ is given by

$$
r_{j^{\prime}}=\max \left\{w\left(i, j^{\prime}\right)-q_{i} \mid i \in N \cup\{0\},\left(i, j^{\prime}\right) \in F \cup\left\{\left(0, j^{\prime}\right)\right\}\right\} \quad\left(j^{\prime} \in N^{\prime}\right) .
$$

To derive the equation (3.9), it suffices to show that for $q \in \mathscr{H}_{0}$ we have

$$
\max \left\{w\left(i, j^{\prime}\right)-q_{i} \mid i \in N,\left(i, j^{\prime}\right) \in F\right\} \geq w\left(0, j^{\prime}\right)-q_{0}=0 \quad\left(\forall j^{\prime} \in N^{\prime}\right) .
$$

Assume that $\eta$ is an equilibrium matching in $\left(N, N^{\prime}, F, w\right)$ that is a perfect matching; such $\eta$ exists by Proposition 3.1. By Proposition 2.6 (iii), $(\eta, q, r)$ is an equilibrium in $\left(N, N^{\prime}, F, w\right)$, and therefore we have (3.7) and (3.8), implying that

$$
w\left(\eta\left(j^{\prime}\right), j^{\prime}\right)-q_{\eta\left(j^{\prime}\right)}=r_{j^{\prime}} \geq 0 \quad\left(\forall j^{\prime} \in N^{\prime}\right) .
$$

Therefore, (3.15) holds.

[Proof of (iii)] By (3.15), the set $\widetilde{D}_{j^{\prime}}(q) \backslash\{0\}$ is nonempty for all $j^{\prime} \in N^{\prime}$. It follows from the proof of (i) and Proposition 2.8 that $\eta$ is an equilibrium matching if and only if $\eta\left(j^{\prime}\right) \in \widetilde{D}_{j^{\prime}}(q)$ holds for all $j^{\prime} \in N^{\prime}$. Since $\eta\left(j^{\prime}\right) \neq 0$, we have $\eta\left(j^{\prime}\right) \in \widetilde{D}_{j^{\prime}}(q)$ if and only if $\eta\left(j^{\prime}\right) \in \widetilde{D}_{j^{\prime}}(q) \backslash\{0\}$.

\subsubsection{Proof of Theorem 3.3}

We first prove the equivalence of (a) and (b), and then the equivalence of (b) and (c).

[Proof of $(\mathrm{a}) \Leftrightarrow(\mathrm{b})] \quad$ Recall that $(\mu, p)$ is an equilibrium in $(N, E, v)$ if and only if it satisfies the conditions (2.5) and (2.6). By the definitions of $F$, 
$w$, and $\eta_{\mu}$, the conditions can be rewritten in terms of the assignment game $\left(N, N^{\prime}, F, w\right)$ as

$$
\begin{array}{ll}
p_{i}+p_{j^{\prime}} \geq w\left(i, j^{\prime}\right) & \left(\forall\left(i, j^{\prime}\right) \in F\right), \\
p_{\eta_{\mu}\left(j^{\prime}\right)}+p_{j^{\prime}}=w\left(\eta_{\mu}\left(j^{\prime}\right), j^{\prime}\right) & \left(\forall j^{\prime} \in N^{\prime}\right) .
\end{array}
$$

The condition (3.16) implies $p_{i} \geq 0$ for $i \in N$ since $2 p_{i}=p_{i}+p_{i^{\prime}} \geq w\left(i, i^{\prime}\right)=0$ for $i \in N$. By Lemma 3.2 (i), the conditions (3.16) and (3.17) hold if and only if $\left(\eta_{\mu}, p, p\right)$ is an equilibrium in $\left(N, N^{\prime}, F, w\right)$.

[Proof of $(b) \Leftrightarrow(c)] \quad$ The implication “(b) $\Rightarrow(c)$ " is easy to see by setting $q=r=p$. The converse can be shown as follows.

By assumption, $\eta_{\mu}$ and $(q, r)$ are an equilibrium matching and an equilibrium payoff in $\left(N, N^{\prime}, F, w\right)$, respectively. Due to the symmetric structure of $\left(N, N^{\prime}, F, w\right)$, we can easily observe that the tuple $(\widehat{\eta}, r, q)$ with the matching $\widehat{\eta}: N^{\prime} \rightarrow N$ given by

$$
\widehat{\eta}\left(j^{\prime}\right)=i \text { if } \eta_{\mu}\left(i^{\prime}\right)=j \quad\left(\forall j^{\prime} \in N^{\prime}\right)
$$

is an equilibrium in $\left(N, N^{\prime}, F, w\right)$. Hence, $(r, q)$ is also an equilibrium payoff in $\left(N, N^{\prime}, F, w\right)$. By Proposition 2.6 (ii), the set of an equilibrium payoffs in $\left(N, N^{\prime}, F, w\right)$ is given as a convex polyhedron. Hence, the payoff $(p, p)=(1 / 2)[(q, r)+(r, q)]$ is also an equilibrium payoff in $\left(N, N^{\prime}, F, w\right)$. By Proposition 2.6 (iii), $\left(\eta_{\mu}, p, p\right)$ is an equilibrium in $\left(N, N^{\prime}, F, w\right)$.

\subsubsection{Proof of Theorem 3.4}

The claim (i) can be shown by the following chain of equivalence:

$\mu$ is an equilibrium matching in $(N, E, v)$

$\Longleftrightarrow(\mu, p)$ is an equilibrium in $(N, E, v)$ for some payoff $p \in \mathbb{R}^{N}$

(by the definition of equilibrium matchings)

$\Longleftrightarrow\left(\eta_{\mu}, q, r\right)$ is an equilibrium in $\left(N, N^{\prime}, F, w\right)$

$$
\text { for some payoff }(q, r) \in \mathbb{R}^{N} \times \mathbb{R}^{N^{\prime}}
$$

(by Theorem 3.3)

$\Longleftrightarrow \eta_{\mu}$ is an equilibrium matching in $\left(N, N^{\prime}, F, w\right)$

(by the definition of equilibrium matchings).

The claim (ii) can be also shown in a similar way. Let $\mu: N \rightarrow N$ be an equilibrium matching in $(N, E, v)$; the existence follows from the assumption 
of the claim (ii). Then, the claim (i) shown above implies that the matching $\eta_{\mu}: N^{\prime} \rightarrow N$ in $\left(N, N^{\prime}, F, w\right)$ given by (3.11) is an equilibrium matching in $\left(N, N^{\prime}, F, w\right)$. Therefore, we obtain the following chain of equivalence:

$p$ is an equilibrium payoff in $(N, E, v)$

$\Longleftrightarrow(\mu, p)$ is an equilibrium in $(N, E, v)$

(by the definition of equilibrium payoffs and Proposition 2.3 (iii))

$\Longleftrightarrow\left(\eta_{\mu}, q, r\right)$ is an equilibrium in $\left(N, N^{\prime}, F, w\right)$ for some payoff

$$
(q, r) \in \mathbb{R}^{N} \times \mathbb{R}^{N^{\prime}} \text { with }(1 / 2)(q+r)=p \quad \text { (by Theorem 3.3) }
$$

$\Longleftrightarrow$ there exists an equilibrium payoff $(q, r) \in \mathbb{R}^{N} \times \mathbb{R}^{N^{\prime}}$

in $\left(N, N^{\prime}, F, w\right)$ such that $(1 / 2)(q+r)=p$

(by the definition of equilibrium payoffs and Proposition 2.6 (iii)).

\subsubsection{Proof of Theorem 3.5}

Let $\eta_{\mu}: N^{\prime} \rightarrow N$ be the matching in $\left(N, N^{\prime}, F, w\right)$ given by (3.11). By Theorem 3.4 (i), $\mu$ is an equilibrium matching in $(N, E, v)$ if and only if $\eta_{\mu}$ is an equilibrium matching in $\left(N, N^{\prime}, F, w\right)$, which is in turn equivalent to the condition

$$
\eta_{\mu}\left(i^{\prime}\right) \in \widetilde{D}_{i^{\prime}}(q) \backslash\{0\} \quad\left(\forall i^{\prime} \in N^{\prime}\right)
$$

by Lemma 3.2 (iii). Since the set $\widetilde{D}_{i^{\prime}}(q) \backslash\{0\}$ is nonempty by Lemma 3.2 (iii), we have $\widetilde{D}_{i^{\prime}}(q) \backslash\{0\}=D_{i}(q)$ for $i \in N$. We also have $\mu(i)=\eta_{\mu}\left(i^{\prime}\right) \neq 0$ for $i \in N$, which implies that the condition (3.18) can be rewritten as $\mu(i) \in$ $D_{i}(q)(\forall i \in N)$. This concludes the proof of (i).

We then prove the claim (ii). Define a vector $r \in \mathbb{R}^{N}$ by

$$
r(i)=v(\mu(i), i)-q_{\mu(i)} \quad(i \in N) .
$$

We will show below that $(q, r)$ is an equilibrium payoff in $\left(N, N^{\prime}, F, w\right)$. Since $p=(1 / 2)(q+r)$ holds, it follows from Theorem 3.4 (ii) that $p$ is an equilibrium payoff in $(N, E, v)$.

We now show that $(q, r)$ is an equilibrium payoff in $\left(N, N^{\prime}, F, w\right)$. Since $\eta_{\mu}\left(i^{\prime}\right)=\mu(i)$ for $i \in N$ and $v(i, j)=w\left(i, j^{\prime}\right)$ for $(i, j) \in E$, the equation (3.19) can be rewritten as

$$
r\left(i^{\prime}\right)=w\left(\eta_{\mu}\left(i^{\prime}\right), i^{\prime}\right)-q_{\eta_{\mu}\left(i^{\prime}\right)} \quad\left(\forall i^{\prime} \in N^{\prime}\right) .
$$


Since $\eta_{\mu}$ is an equilibrium matching in $\left(N, N^{\prime}, F, w\right)$, the condition (3.18) follows from the proof of the claim (i). Hence, we have

$$
\begin{aligned}
r\left(i^{\prime}\right) & =w\left(\eta_{\mu}\left(i^{\prime}\right), i^{\prime}\right)-q_{\eta_{\mu}\left(i^{\prime}\right)} \\
& =\max \left\{w\left(i, j^{\prime}\right)-q_{i} \mid i \in N,\left(i, j^{\prime}\right) \in F\right\} \quad\left(\forall i^{\prime} \in N^{\prime}\right) .
\end{aligned}
$$

Since $q \in \mathscr{H}_{0}$, this equation and Lemma 3.2 (ii) imply that $(q, r)$ is an equilibrium payoff in $\left(N, N^{\prime}, F, w\right)$.

\section{CONNECTION TO THE ALGORITHM OF ANDERSSON ET AL.}

\subsection{Theorems}

We consider the algorithm by Andersson et al. (2014b) for finding an equilibrium in the partnership formation problem, and discuss the connection with our algorithm COMPUTEEQUILIBRIUM. The difference between the two algorithms is in the choice of a vector $q$; while $q$ is selected from the set $\mathscr{H}_{0}$ in our algorithm, it is computed by a certain price adjustment process in the algorithm by Andersson et al. (2014b). In the following, we show that the vector $q$ used in Andersson et al. (2014b) is the unique minimal vector in $\mathscr{H}_{0}$. This implies that the algorithm in Andersson et al. (2014b) can be viewed as a specific implementation of our algorithm. We also discuss the relationship between the price adjustment process used in the algorithm of Andersson et al. (2014b) and the algorithm VICKREYENGLISH for the assignment game in Section 2.2.

We first explain the algorithm by Andersson et al. (2014b). In this section, we assume that the values $v(i, j)$ are integers, as in Andersson et al. (2014b). For a payoff $p \in \mathbb{R}^{N}$ and a set $S \subseteq N$ of agents, we denote by $O(S, p)$ the set of agents $j \in N$ such that all of $j$ 's best (possible) partners under the payoff $p$ are contained in the set $S$, and by $U(S, p)$ the set of agents $j \in N$ such that at least one of $j$ 's best (possible) partners of $j$ under the payoff $p$ is contained in $S$. That is,

$$
\begin{aligned}
& O(S, p)=\left\{j \in N \mid D_{j}(p) \subseteq S\right\}, \\
& U(S, p)=\left\{j \in N \mid D_{j}(p) \cap S \neq \emptyset\right\} .
\end{aligned}
$$

A set $S \subseteq N$ is said to be overdemanded if $|O(S, p)|>|S|$, and underdemanded if $|U(S, p)|<|S|$. If some set $S \subseteq N$ is overdemanded, then it is impossible 
to assign distinct partners in $S$ to all agents in $O(S, p)$, implying that there exists no matching $\mu: N \rightarrow N$ such that $\mu(i) \in D_{i}(p)(\forall i \in N)$. Similarly, if an underdemanded set exists, then there exists no matching $\mu$ with $\mu(i) \in D_{i}(p)$ $(\forall i \in N)$.

We say that $S$ is in excess demand at payoff $p$ if the following condition holds:

$$
|U(T, p) \cap O(S, p)|>|T| \quad(\emptyset \neq \forall T \subsetneq S) .
$$

It is known (see Andersson et al. (2010, 2013); Mo et al. (1988)) that a set in excess demand exists if an overdemanded set exists (i.e., $|O(S, p)|>|S|$ holds for some $S \subseteq N$ ), and a maximal set in excess demand is uniquely determined.

We describe below the algorithm by Andersson et al. (2014b) in its variant given in Andersson et al. (2014a). The difference between the algorithm PARTNERSHIPFORMATION and our algorithm is only in the choice of a vector $q$ in Step 1.

\section{Algorithm PARTNERSHIPFORMATION}

Step 1: Compute a vector $q \in \mathbb{R}^{N}$ by the algorithm PROCESSAEGHK given below.

Step 2: If there exists no matching $\mu: N \rightarrow N$ in $(N, E, v)$ satisfying (3.12), then assert that "no equilibrium exists in $(N, E, v)$ " and stop.

Step 3: Find a matching $\mu: N \rightarrow N$ in $(N, E, v)$ satisfying (3.12), and let $p \in \mathbb{R}^{N}$ be a vector given by (3.13).

Output $(\mu, p)$ as an equilibrium of $(N, E, v)$.

\section{Algorithm PROCESSAEGHK}

Step 0: Set $q \in \mathbb{Z}^{N}$ by $q:=(0,0, \ldots, 0)$.

Step 1: Collect the demand correspondences $D_{j}(q)$ for $j \in N$.

Step 2: If $|O(S, q)| \leq|S|$ holds for every $S \subseteq N$, then output $q$ and stop.

Step 3: Find the unique maximal set $S^{*} \subseteq N$ in excess demand at payoff $q$, update $q$ by $q_{i}:=q_{i}+1\left(i \in S^{*}\right)$, and go to Step 1 .

We denote by $q^{*}$ the output of the algorithm ProcESSAEGHK, i.e., $q^{*}$ is the vector $q$ used in PARTNERSHIPFORMATION. We also denote by $\widehat{q}$ the unique minimal vector in $\mathscr{H}_{0}$. We will show that $q^{*}=\widehat{q} \in \mathscr{H}_{0}$ holds. Hence, the algorithm PROCESSAEGHK can be seen as a specific implementation of Step 1 in our algorithm.

Theorem 4.1. The vector $q^{*}$ found in the algorithm PROCESSAEGHK is equal to the unique minimal vector $\widehat{q}$ in $\mathscr{H}_{0}$. 
Proof. Proof is given in Section 4.2.1.

We then show that the trajectory of the vector $q$ in the algorithm PROCESSAEGHK is the same as that of the algorithm VICKREYENGLISH applied to the associated assignment game $\left(N, N^{\prime}, F, w\right)$. It should be noted that the algorithms PROCESSAEGHK and VICKREYENGLISH use different kind of demand correspondences; the former uses $D_{i}(q) \subseteq N$, while the latter uses $\widetilde{D}_{j^{\prime}}(q) \subseteq N \cup\{0\}$.

Theorem 4.2. Suppose that the algorithm PROCESSAEGHK is applied to the partnership formation problem $(N, E, v)$. Then, the trajectory of the vector $q$ is the same as that of the algorithm VICKREYENGLISH applied to the associated assignment game $\left(N, N^{\prime}, F, w\right)$.

Proof. Proof is given in Section 4.2.2.

\subsection{Proofs}

\subsubsection{Proof of Theorem 4.1}

Two lemmas are given before we start the proof of Theorem 4.1. We define

$$
\mathscr{H}=\left\{q \in \mathbb{R}^{N}\left|q_{i} \geq 0(\forall i \in N),\right| O(S, q)|\leq| S \mid(\forall S \subseteq N)\right\} .
$$

This set is used in Andersson et al. (2014b) to prove the validity of their algorithm. In particular, the following fact is proved.

Lemma 4.3 (Andersson et al. (2014b, Theorem 2)). A minimal vector in $\mathscr{H}$ is uniquely determined and equal to the vector $q^{*}$.

We will prove that a vector $q$ is in the set $\mathscr{H}_{0}$ if and only if $q$ is a vector in $\mathscr{H}$ satisfying the additional condition that

$$
\max \left\{w\left(i, j^{\prime}\right)-q_{i} \mid i \in N,\left(i, j^{\prime}\right) \in F\right\} \geq 0 \quad\left(\forall j^{\prime} \in N^{\prime}\right) .
$$

This additional condition means that the payoff of every buyer $j^{\prime} \in N^{\prime}$ in the associated assignment game $\left(N, N^{\prime}, F, w\right)$ is non-negative under the prices given by $q$.

Lemma 4.4. The set $\mathscr{H}_{0} \subseteq \mathbb{R}^{N}$ given by (3.5) can be rewritten as

$$
\mathscr{H}_{0}=\{q \in \mathscr{H} \mid q \text { satisfies (4.2) }\} \text {. }
$$


Proof. For $q \in \mathbb{R}^{N}$, we define a vector $r \in \mathbb{R}^{N^{\prime}}$ by

$$
r_{j^{\prime}}=\max \left\{w\left(i, j^{\prime}\right)-q_{i} \mid i \in N,\left(i, j^{\prime}\right) \in F\right\} \quad\left(j^{\prime} \in N^{\prime}\right) .
$$

To prove the equation (4.3), we will show the following equivalence:

$$
q \in \mathscr{H}_{0} \quad \text { if and only if } \quad q \in \mathscr{H} \text { and } r_{j^{\prime}} \geq 0 \text { for all } j^{\prime} \in N^{\prime} .
$$

We first prove the "only if" part of this equivalence, and then the "if" part.

[Proof of "only if" part] Let $q \in \mathscr{H}_{0}$. Then, the pair $(q, r)$ is an equilibrium payoff in the associated assignment game $\left(N, N^{\prime}, F, w\right)$ by Lemma 3.2 (ii), and therefore we have

$$
q_{i} \geq 0(\forall i \in N), \quad r_{j^{\prime}} \geq 0\left(\forall j^{\prime} \in N^{\prime}\right) .
$$

To prove $q \in \mathscr{H}$, we need to show that $|O(S, q)| \leq|S|$ holds for every $S \subseteq N$ since $q_{i} \geq 0$ holds for all $i \in N$. Let $\eta: N^{\prime} \rightarrow N$ be an equilibrium matching in the associated assignment game $\left(N, N^{\prime}, F, w\right)$ such that $\eta$ is a perfect matching. Since $q \in \mathscr{H}_{0}$, we have $\eta\left(j^{\prime}\right) \in \widetilde{D}_{j^{\prime}}(q) \backslash\{0\}=D_{j}(q)$ for $j^{\prime} \in N^{\prime}$ by Lemma 3.2 (iii). Therefore, it holds that

$$
|O(S, q)|=\left|\left\{\eta\left(j^{\prime}\right) \mid j \in O(S, q)\right\}\right| \leq\left|\bigcup_{j \in O(S, q)} D_{j}(q)\right| \leq|S|,
$$

where the last inequality is by $D_{j}(q) \subseteq S$ for $j \in O(S, q)$.

[Proof of "if" part] Suppose that $q \in \mathscr{H}$ and $r_{j^{\prime}} \geq 0$ for all $j^{\prime} \in N^{\prime}$. To prove $q \in \mathscr{H}_{0}$, it suffices to show that there exists a perfect matching $\eta: N^{\prime} \rightarrow N$ in the associated assignment game $\left(N, N^{\prime}, F, w\right)$ such that $(\eta, q, r)$ is an equilibrium in $\left(N, N^{\prime}, F, w\right)$.

For every $j^{\prime} \in N^{\prime}$, we have $\widetilde{D}_{j^{\prime}}(q) \backslash\{0\}=D_{j}(q)$ since

$$
\max \left\{w\left(i, j^{\prime}\right)-q_{i} \mid i \in N,\left(i, j^{\prime}\right) \in F\right\}=r_{j^{\prime}} \geq 0 .
$$

It follows that for every $S \subseteq N$,

$$
\widetilde{U}(S, q)=\left\{j^{\prime} \in N^{\prime} \mid \widetilde{D}_{j^{\prime}}(q) \cap S \neq \emptyset\right\}=\left\{j \in N \mid D_{j}(q) \cap S \neq \emptyset\right\}=U(S, q) .
$$

We also have $U(S, q)=N \backslash O(N \backslash S, q)$. Hence, it holds that

$$
|\widetilde{U}(S, q)|=|U(S, q)|=|N|-|O(N \backslash S, q)| \geq|N|-|N \backslash S|=|S|,
$$


where the inequality holds by $q \in \mathscr{H}$. By the well-known Hall's theorem in graph theory (see, e.g., Schrijver (2003)), the condition $|\widetilde{U}(S, q)| \geq|S|(\forall S \subseteq$ $N)$ implies the existence of a perfect matching $\eta: N^{\prime} \rightarrow N$ in $\left(N, N^{\prime}, F, w\right)$ such that $\eta\left(j^{\prime}\right) \in \widetilde{D}_{j^{\prime}}(q) \backslash\{0\}\left(\forall j^{\prime} \in N^{\prime}\right)$. Then, it is not difficult to see that $(\eta, q, r)$ satisfies the conditions (3.6), (3.7), and (3.8), i.e., $(\eta, q, r)$ is an equilibrium by Lemma 3.2 (i). Thus, we have $q \in \mathscr{H}_{0}$ by the definition of $\mathscr{H}_{0}$.

We now prove Theorem 4.1.

Proof of Theorem 4.1. By Lemma 4.4, it holds that $\widehat{q} \in \mathscr{H}_{0} \subseteq \mathscr{H}$. This implies that $q^{*} \leq \widehat{q}$ since $q^{*}$ is the unique minimal vector in $\mathscr{H}$. Therefore, for every $j^{\prime} \in N^{\prime}$ it holds that

$$
\begin{aligned}
& \max \left\{w\left(i, j^{\prime}\right)-q_{i}^{*} \mid i \in N,\left(i, j^{\prime}\right) \in F\right\} \\
& \geq \max \left\{w\left(i, j^{\prime}\right)-\widehat{q}_{i} \mid i \in N,\left(i, j^{\prime}\right) \in F\right\} \geq 0,
\end{aligned}
$$

where the last inequality is by $\widehat{q} \in \mathscr{H}$. Hence, we have $q^{*} \in \mathscr{H}_{0}$ by Lemma 4.4. From the minimality of $\widehat{q}$ in $\mathscr{H} 0$, we have $q^{*} \geq \widehat{q}$, implying that $q^{*}=\widehat{q}$.

\subsubsection{Proof of Theorem 4.2}

We assume that the trajectory of the vector $q$ is the same until the $k$-th iteration of the two algorithms, and show that $q$ remains the same in the $(k+1)$-st iteration. For this, we will prove that the set $S^{*}$ in Step 3 of the $k$-th iteration in PRocessAeGHK is the same as the set $\widetilde{S}^{*}$ in Step 2 of the $k$-th iteration in VicKREYENGLISH. Recall that $S^{*}$ and $\widetilde{S}^{*}$ are defined by using demand correspondences of different kinds.

We consider the vector $q$ at the beginning of the $k$-th iteration in PROCESSAEGHK (and in VICKREYENGLISH). Since $q^{*}$ and $\widehat{q}$ are outputs of PROCESSAEGHK and VICKREYENGLISH, respectively, we have $q \leq q^{*}=\widehat{q}$, where the equality is by Theorem 4.1.

To prove $\widetilde{S}^{*}=S^{*}$, we show that the following conditions hold:

$$
\begin{aligned}
& \widetilde{D}_{j^{\prime}}(q) \backslash\{0\}=D_{j}(q) \quad(\forall j \in N), \\
& \widetilde{U}(Y, q)=U(Y, q) \quad(\forall Y \subseteq N) . \\
& \widetilde{O}\left(\widetilde{S}^{*}, q\right)=O\left(\widetilde{S}^{*}, q\right), \\
& \widetilde{O}\left(S^{*}, q\right)=O\left(S^{*}, q\right) .
\end{aligned}
$$


The proofs of (4.5)-(4.8) are given later.

We first show that $\widetilde{S}^{*} \subseteq S^{*}$ holds. Since $S^{*}$ is the maximal set in excess demand with respect to the demand correspondences $D_{j}(q)$, it suffices to show that $\widetilde{S}^{*}$ is a set in excess demand with respect to the demand correspondences $D_{j}(q)$. For each $Y$ with $\emptyset \neq Y \subseteq \widetilde{S}^{*}$, we have

$$
\left|U(Y, q) \cap O\left(\widetilde{S}^{*}, q\right)\right|=\left|\widetilde{U}(Y, q) \cap \widetilde{O}\left(\widetilde{S}^{*}, q\right)\right|>|Y|,
$$

where the equality is by (4.6) and (4.7), and the inequality is by the definition of $\widetilde{S}^{*}$. This shows that $\widetilde{S}^{*}$ is a set in excess demand with respect to the demand correspondences $D_{j}(q)$. By the maximality of $S^{*}$, we have $\widetilde{S}^{*} \subseteq S^{*}$.

Since $\widetilde{S}^{*}$ is the maximal set in excess demand with respect to the demand correspondences $\widetilde{D}_{j}(q)$, we can show the inclusion $\widetilde{S}^{*} \supseteq S^{*}$ in the same way as above by using (4.6) and (4.8). Hence, we have $\widetilde{S}^{*}=S^{*}$.

To conclude the proof, we show that the conditions (4.5)-(4.8) hold.

We first prove (4.5). Since $q \leq \widehat{q}$, we have

$$
\begin{aligned}
& \max \left\{w\left(i, j^{\prime}\right)-q_{i} \mid i \in N,\left(i, j^{\prime}\right) \in F\right\} \\
& \geq \max \left\{w\left(i, j^{\prime}\right)-\widehat{q}_{i} \mid i \in N,\left(i, j^{\prime}\right) \in F\right\} \geq 0 \quad\left(\forall j^{\prime} \in N^{\prime}\right),
\end{aligned}
$$

where the last inequality is by $\widehat{q} \in \mathscr{H}_{0}$ and Lemma 4.4. Therefore, we have

$$
\begin{aligned}
\widetilde{D}_{j^{\prime}}(q) & =\arg \max \left\{w\left(i, j^{\prime}\right)-q_{i} \mid i \in N \cup\{0\},\left(i, j^{\prime}\right) \in F \cup\left\{\left(0, j^{\prime}\right)\right\}\right\} \\
& \supseteq \arg \max \left\{w\left(i, j^{\prime}\right)-q_{i} \mid i \in N,\left(i, j^{\prime}\right) \in F\right\} \\
& =\arg \max \left\{v(i, j)-q_{i} \mid i \in N,(i, j) \in \bar{E}\right\}=D_{j}(q)
\end{aligned}
$$

for $j \in N$, from which (4.5) follows.

The equation (4.6) can be obtained from (4.5) as follows:

$$
\widetilde{U}(Y, q)=\left\{j^{\prime} \in N^{\prime} \mid \widetilde{D}_{j^{\prime}}(q) \cap Y \neq \emptyset\right\}=\left\{j \in N \mid D_{j}(q) \cap Y \neq \emptyset\right\}=U(Y, q) .
$$

We finally prove (4.7) and (4.8). By (4.5), we have $D_{j}(q) \subseteq \widetilde{D}_{j^{\prime}}(q)$ for all $j \in N$, from which follows that

$$
\widetilde{O}(Y, q)=\left\{j^{\prime} \in N^{\prime} \mid \widetilde{D}_{j^{\prime}}(q) \subseteq Y\right\} \subseteq\left\{j \in N \mid D_{j}(q) \subseteq Y\right\}=O(Y, q) .
$$

for every $Y \subseteq N$. Hence, it suffices to show that

$$
O\left(\widetilde{S}^{*}, q\right) \subseteq \widetilde{O}\left(\widetilde{S}^{*}, q\right), \quad O\left(S^{*}, q\right) \subseteq \widetilde{O}\left(S^{*}, q\right)
$$


In the following, we prove the latter only; the former can be proven similarly.

To prove the inclusion $O\left(S^{*}, q\right) \subseteq \widetilde{O}\left(S^{*}, q\right)$, we show that $j^{\prime} \in \widetilde{O}\left(S^{*}, q\right)$ holds for every $j \in O\left(S^{*}, q\right)$.

Let $j \in O\left(S^{*}, q\right)$. Then, $D_{j}(q) \subseteq S^{*}$ holds by the definition of $O\left(S^{*}, q\right)$. If $0 \notin \widetilde{D}_{j^{\prime}}(q)$, then (4.5) implies that $\widetilde{D}_{j^{\prime}}(q)=D_{j}(q) \subseteq S^{*}$, i.e., $j^{\prime} \in \widetilde{O}\left(S^{*}, q\right)$ holds. We assume, to the contrary, that $0 \in \widetilde{D}_{j^{\prime}}(q)$ and derive a contradiction.

Since $0 \in \widetilde{D}_{j^{\prime}}(q)$ and $\widetilde{D}_{j^{\prime}}(q) \backslash\{0\}=D_{j}(q) \neq \emptyset$, we have

$$
\max \left\{w\left(i, j^{\prime}\right)-q_{i} \mid i \in N,\left(i, j^{\prime}\right) \in F\right\}=0 .
$$

Let $\bar{q} \in \mathbb{Z}^{N}$ be a vector given by

$$
\bar{q}_{i}=\left\{\begin{array}{ll}
q_{i}+1 & \left(\text { if } i \in S^{*}\right), \\
q_{i} & \text { (otherwise) }
\end{array} \quad(i \in N) .\right.
$$

That is, $\bar{q}$ is the vector $q$ in the $(k+1)$-st iteration in PROCESSAEGHK. Since $q \leq \bar{q} \leq q^{*}=\widehat{q}$, we have

$$
\begin{aligned}
0 & =\max \left\{w\left(i, j^{\prime}\right)-q_{i} \mid i \in N,\left(i, j^{\prime}\right) \in F\right\} \\
& \geq \max \left\{w\left(i, j^{\prime}\right)-\bar{q}_{i} \mid i \in N,\left(i, j^{\prime}\right) \in F\right\} \\
& \geq \max \left\{w\left(i, j^{\prime}\right)-\widehat{q}_{i} \mid i \in N,\left(i, j^{\prime}\right) \in F\right\} \geq 0,
\end{aligned}
$$

where the equality is by (4.10) and the last inequality is by (4.9). Hence, we have

$$
\max \left\{w\left(i, j^{\prime}\right)-\bar{q}_{i} \mid i \in N,\left(i, j^{\prime}\right) \in F\right\}=\max \left\{w\left(i, j^{\prime}\right)-q_{i} \mid i \in N,\left(i, j^{\prime}\right) \in F\right\} .
$$

It holds that

$$
\begin{aligned}
& \max \left\{w\left(i, j^{\prime}\right)-\bar{q}_{i} \mid i \in S^{*},\left(i, j^{\prime}\right) \in F\right\} \\
& =\max \left\{w\left(i, j^{\prime}\right)-\left(q_{i}+1\right) \mid i \in S^{*},\left(i, j^{\prime}\right) \in F\right\} \\
& =\max \left\{w\left(i, j^{\prime}\right)-q_{i} \mid i \in S^{*},\left(i, j^{\prime}\right) \in F\right\}-1 \\
& \leq \max \left\{w\left(i, j^{\prime}\right)-q_{i} \mid i \in N,\left(i, j^{\prime}\right) \in F\right\}-1 .
\end{aligned}
$$

We also have

$$
\begin{aligned}
& \max \left\{w\left(i, j^{\prime}\right)-\bar{q}_{i} \mid i \in N \backslash S^{*},\left(i, j^{\prime}\right) \in F\right\} \\
& =\max \left\{w\left(i, j^{\prime}\right)-q_{i} \mid i \in N \backslash S^{*},\left(i, j^{\prime}\right) \in F\right\} \\
& \leq \max \left\{w\left(i, j^{\prime}\right)-q_{i} \mid i \in N,\left(i, j^{\prime}\right) \in F\right\}-1,
\end{aligned}
$$


where the inequality is by the facts that $\widetilde{D}_{j^{\prime}}(q) \backslash\{0\} \subseteq S^{*}$ and $w\left(i, j^{\prime}\right)$ and $q_{i}$ are integer-valued. It follows from (4.12) and (4.13) that

$$
\begin{aligned}
& \max \left\{w\left(i, j^{\prime}\right)-\bar{q}_{i} \mid i \in N,\left(i, j^{\prime}\right) \in F\right\} \\
& =\max \left[\max \left\{w\left(i, j^{\prime}\right)-\bar{q}_{i} \mid i \in S^{*},\left(i, j^{\prime}\right) \in F\right\},\right. \\
& \left.\quad \max \left\{w\left(i, j^{\prime}\right)-\bar{q}_{i} \mid i \in N \backslash S^{*},\left(i, j^{\prime}\right) \in F\right\}\right] \\
& \leq \max \left\{w\left(i, j^{\prime}\right)-q_{i} \mid i \in N,\left(i, j^{\prime}\right) \in F\right\}-1 \\
& <\max \left\{w\left(i, j^{\prime}\right)-q_{i} \mid i \in N,\left(i, j^{\prime}\right) \in F\right\},
\end{aligned}
$$

a contradiction to (4.11). This concludes the proof of (4.8).

\section{A. APPENDIX: REVIEW OF PRICE ADJUSTMENT PROCESSES FOR ASSIGNMENT GAMES}

We review price adjustment processes for the assignment game $(A, B, F, w)$. A price adjustment process is an algorithm (a mechanism, more precisely) for finding an equilibrium price vector of the assignment game (i.e., a vector in $\mathscr{H}_{0}$ ) by iteratively updating the price vector, where the information of buyers' demand correspondences are used. We have already presented the algorithm VICKREYENGLISH in Section 2.2 as an example of a price adjustment process. We present some other price adjustment processes based on Lyapunov function minimization.

For buyer $j \in B$, we define the indirect utility function $V_{j}: \mathbb{R}_{+}^{A} \rightarrow \mathbb{R}$ by

$$
V_{j}(q)=\max \left\{w(i, j)-q_{i} \mid i \in A \cup\{0\}\right\} \quad\left(q \in \mathbb{R}_{+}^{A}\right) .
$$

We also define the Lyapunov function $L: \mathbb{R}^{A} \rightarrow \mathbb{R}$ by

$$
L(q)=\sum_{j \in B} V_{j}(q)+\sum_{i \in A} q_{i} \quad\left(q \in \mathbb{R}_{+}^{A}\right) .
$$

Throughout this section, we assume that the values $w(i, j)((i, j) \in F)$ are non-negative integers. It is known (Ausubel (2006), Murota et al. (2016)) that the set of minimizers of the Lyapunov function $L$ coincides with the set of equilibrium price vectors in the assignment game. Moreover, the integrality of values $w(i, j)$ implies the existence of an integral minimizer of the Lyapunov function $L$; in particular, the unique minimal and maximal minimizers of the Lyapunov function $L$ are integral vectors. Based on this fact, we can compute 
an equilibrium price vector by finding an integral minimizer of the Lyapunov function $L$.

The following ascending-type price adjustment process finds a minimizer of the Lyapunov function (Ausubel (2006)). For a set $S \subseteq A$, we denote by $\chi_{S} \in\{0,1\}^{A}$ the characteristic vector of $S$, i.e.,

$$
\left(\chi_{S}\right)_{i}= \begin{cases}1 & (\text { if } i \in S) \\ 0 & \text { (otherwise) }\end{cases}
$$

\section{Algorithm AscendMinimaL}

Step 0: Set $q:=q^{\circ}$, where $q^{\circ} \in \mathbb{Z}_{+}^{A}$ is an arbitrary vector that is

a lower bound of the minimal vector in $\mathscr{H}_{0}$ (e.g., $q^{\circ}=(0,0, \ldots, 0)$ ).

Step 1: Collect the demand correspondences $\widetilde{D}_{j}(q)$ for $j \in B$.

Step 2: Find the unique minimal set $S \subseteq A$ minimizing $L\left(q+\chi_{S}\right)$.

Step 3: If $L\left(q+\chi_{S}\right)=L(q)$, then output $q$ and stop.

Step 4: Update $q$ by $q:=q+\chi_{S}$, and go to Step 1 .

It should be noted that computation of a set $S \subseteq A$ minimizing $L\left(q+\chi_{S}\right)$ in Step 2 can be done by using the demand correspondences $\widetilde{D}_{j}(q)(j \in B)$ since it holds that

$$
L\left(q+\chi_{S}\right)-L(q)=|S|-|\widetilde{O}(S, q)| \quad(S \subseteq A) .
$$

It is shown in Murota et al. (2016) that the behavior of the algorithm ASCENDMINIMAL is exactly the same as the algorithm VICKREYENGLISH in Section 2.2. In particular, the set $S$ computed in Step 2 of each iteration is equal to the the maximal set in excess demand at payoff $q$.

We consider a variant of the algorithm ASCENDMINIMAL, called ASCENDMAXIMAL, where the initial vector $q^{\circ}$ is chosen to be a lower bound of the unique maximal vector in $\mathscr{H}_{0}$ in Step 0 , and the unique maximal set $S \subseteq A$ minimizing $L\left(q+\chi_{S}\right)$ is used in Step 2 .

Each of the algorithms AsCENDMINIMAL and ASCENDMAXIMAL finds an equilibrium price vector of the assignment game. Moreover, ASCENDMINIMAL (resp., ASCENDMAXIMAL) finds the unique minimal (resp., maximal) equilibrium price vector.

Theorem A.1 (Ausubel (2006); Murota et al. (2016)). For the assignment game $(A, B, F, w)$, the algorithm ASCENDMINIMAL (resp., AsCENDMAXIMAL) outputs the unique minimal (resp., maximal) price vector in the set $\mathscr{H}_{0}$ given by (2.19). 
We then present a descending-type price adjustment process, where the price vector decreases monotonically in each iteration.

Algorithm DescendMAXIMAL

Step 0: Set $q:=q^{\circ}$, where $q^{\circ} \in \mathbb{Z}_{+}^{A}$ is an arbitrary vector that is an upper bound of the maximal vector in $\mathscr{H}_{0}$.

Step 1: Collect the demand correspondences $\widetilde{D}_{j}(q)$ for $j \in B$.

Step 2: Find the unique minimal set $S \subseteq A$ minimizing $L\left(q-\chi_{S}\right)$.

Step 3: If $L\left(q-\chi_{S}\right)=L(q)$, then output $q$ and stop.

Step 4: Update $q$ by $q:=q-\chi_{S}$, and go to Step 1 .

We also consider a variant, called DESCENDMINIMAL, where the initial vector $q^{\circ}$ is chosen to be an upper bound of the unique minimal vector in $\mathscr{H}_{0}$ in Step 0, and the unique maximal set $S \subseteq A$ minimizing $L\left(q-\chi_{S}\right)$ is used in Step 2. It is shown in Murota et al. (2016) that the behavior of DESCENDMINIMAL coincides with that of the descending-type price adjustment process due to Mishra \& Parkes (2009).

Theorem A.2 (Ausubel (2006); Murota et al. (2016)). For the assignment game $(A, B, F, w)$, the algorithm DESCENDMINIMAL (resp., DESCENDMAXIMAL) outputs the unique minimal (resp., maximal) price vector in the set $\mathscr{H}_{0}$ given by (2.19).

We finally present the so-called two-phase algorithms for finding an equilibrium (see Murota et al. (2016); see also Murota (2016)). A two-phase algorithm consists of the ascending phase and the descending phase, and the price vector is increasing in the ascending phase and then decreasing in the descending phase. That is, a two-phase algorithm is a combination of two price adjustment processes of ascending-type and descending-type. An important merit of two-phase algorithms is that any price vector can be used as an initial vector, which is impossible in ascending- or descending-type adjustment processes. This flexibility enables us to reduce the number of iterations by selecting an initial vector that is close to an equilibrium price vector.

By the combination of ASCENDMINIMAL/ASCENDMAXIMAL and DESCENDMINIMAL/DESCENDMAXIMAL, we can obtain four variants of twophase algorithms. For example, the combination of ASCENDMINIMAL and DESCENDMAXIMAL yields the following algorithm (Murota (2016); Murota et al. (2016)).

\section{Algorithm TwoPhaseMinMax}

Step 0: Set $q:=q^{\circ}$, where $q^{\circ} \in \mathbb{Z}_{+}^{A}$ is an arbitrary vector. 
Go to Ascending Phase.

Ascending Phase:

Step A1: Collect the demand correspondences $\widetilde{D}_{j}(q)$ for $j \in B$.

Step A2: Find the unique minimal set $S \subseteq A$ minimizing $L\left(q+\chi_{S}\right)$.

Step A3: If $L\left(q+\chi_{S}\right)=L(q)$, then go to Descending Phase.

Step A4: Update $q$ by $q:=q+\chi_{S}$, and go to Step A1.

Descending Phase:

Step D1: Collect the demand correspondences $\widetilde{D}_{j}(q)$ for $j \in B$.

Step D2: Find the unique minimal set $S \subseteq A$ minimizing $L\left(q-\chi_{S}\right)$.

Step D3: If $L\left(q-\chi_{S}\right)=L(q)$, then output $q$ and stop.

Step D4: Update $q$ by $q:=q-\chi_{S}$, and go to Step D1.

The ascending phase stops at the vector $\hat{q}$ that is the unique minimal minimizer of the Lyapunov function in the region $\left\{q \mid q \geq q^{\circ}\right\}$, and the descending phase stops at the vector $\check{q}$ that is the unique maximal minimizer of the Lyapunov function in the region $\{q \mid q \leq \hat{q}\}$, which turns out to be a global minimizer of the Lyapunov function, i.e., an equilibrium price vector (Murota (2016); Murota et al. (2016)).

Theorem A.3 (Murota (2016); Murota et al. (2016)). For the assignment game $(A, B, F, w)$, the algorithm TWOPHASEMINMAX outputs a vector in the set $\mathscr{H}_{0}$ given by (2.19).

The algorithm TwOPHASEMINMAX has the following characteristics:

- TwOPHASEMinMax terminates in a smaller number of iterations than the other three variants of the two-phase algorithms.

- The output of TwOPHASEMINMAX is neither of minimal and maximal equilibrium price vectors in general.

We finally discuss bounds for the number of iterations required by the algorithms explained above. It can be shown that the worst case bound for the number of iterations required by the algorithms explained above is proportional to $\max \{w(i, j) \mid i \in A, j \in B\}$ (see, e.g., Andersson \& Erlanson (2013); Murota (2016); Murota et al. (2016)). This means that the number of iterations required by the algorithms is pseudo-polynomial in the terminology of algorithm theory. 


\section{References}

Alkan, A., \& Tuncay, A. (2013). Pairing games and markets. Working Paper, Sabanci University.

Andersson, T., Andersson, C., \& Talman, A. J. J. (2010). Sets in excess demand in simple ascending auctions with unit-demand bidders. Working Paper, Department of Economics, Lund University, No. 2010:15 (revised June 2012).

Andersson, T., Andersson, C., \& Talman, A. J. J. (2013). Sets in excess demand in simple ascending auctions with unit-demand bidders. Annals of Operations Research, 211, 27-36.

Andersson, T., \& Erlanson, A. (2013). Multi-item Vickrey-English-Dutch auctions. Games and Economic Behavior, 81.

Andersson, T., Gudmundsson, J., Habis, H., Ingerbretsen Carlson, J., \& Kratz, J. (2014a). A method for finding the maximal set in excess demand. Economic Letters, 125, 18-20.

Andersson, T., Gudmundsson, J., Talman, D., \& Yang, Z. (2014b). A competitive partnership formation process. Games and Economics Behavior, 86, 165-177.

Ausubel, L. M. (2006). An efficient dynamic auction for heterogeneous commodities. American Economic Review, 96, 602-629.

Biró, P., Kern, W., \& Paulusma, D. (2012). Computing solutions for matching games. International Journal of Game Theory, 41, 75-90.

Chiappori, P.-A., Galichon, A., \& Salanié, B. (2014). The roommate problem is more stable than you think. CESifo Working Paper Series No. 4676, February, 2014.

Crawford, V. P., \& Knoer, E. M. (1981). Job matching with heterogeneous firms and workers. Econometrica, 49, 437-450.

Demange, G., Gale, D., \& Sotomayor, M. (1986). Multi-item auctions. Journal of Political Economy, 94, 863-872.

Eriksson, K., \& Karlander, J. (2001). Stable outcomes of the roommate game with transferable utility. International Journal of Game Theory, 29, 555-569.

Klaus, B., \& Nichifor, A. (2010). Consistency in one-sided assignment problems. Social Choice and Welfare, 35, 415-433.

Koopmans, T. C., \& Beckmann, M. (1957). Assignment problems and the location of economic activities. Econometrica, 25, 53-76.

Mishra, D., \& Parkes, D. C. (2009). Multi-item Vickrey-Dutch auctions. Games and Economic Behavior, 66, 326-347.

Mo, J.-P., Tsai, P.-S., \& Lin, S.-C. (1988). Pure and minimal overdemanded sets: a note on Demange, Gale, and Sotomayor. Discussion Paper, Institute of Economics, Academia Sinica, Taipei, Taiwan, No. 8808.

Murota, K. (2016). Discrete convex analysis: A tool for economics and game theory. 
Journal of Mechanism and Institution Design, 1, 151-273.

Murota, K., Shioura, A., \& Yang, Z. (2016). Time bounds for iterative auctions: a unified approach by discrete convex analysis. Discrete Optimization, 19, 36-62.

Nemhauser, G. L., \& Trotter, L. E. (1975). Vertex packings: structural properties and algorithms. Mathematical Programming, 8, 232-248.

Pulleyblank, W. R. (1987). Fractional matchings and the Edmonds-Gallai theorem. Discrete Applied Mathematics, 16, 51-58.

Roth, A. E., \& Sotomayor, M. A. O. (1990). Two-Sided Matching: A Study in Game-Theoretic Modeling and Analysis. Cambridge: Cambridge University Press.

Sankaran, J. K. (1994). On a dynamic auction mechanism for a bilateral assignment problem. Mathematical Social Sciences, 28, 143-150.

Schrijver, A. (2003). Combinatorial Optimization: Polyhedra and Efficiency. Berlin: Springer.

Shapley, L. S., \& Shubik, M. (1971). The assignment game I: The core. International Journal of Game Theory, 1, 111-130.

Talman, D., \& Yang, Z. (2011). A model of partnership formation. Journal of Mathematical Econoics, 47, 206-212. 